\title{
Superior semicircular canal dehiscence: The neglected pathology
}

\author{
Hilda G. Guzman-Perez' , Alejandro Quiroga-Garza', José L. Treviño-Gonzalez², \\ Rodrigo E. Elizondo-Omaña ${ }^{1}$, and Santos Guzman-Lopez ${ }^{1}$ \\ ${ }^{1}$ Department of Human Anatomy, School of Medicine; ${ }^{2}$ Department of Otorhinolaryngology, "Dr. José Eleuterio González" University Hospital. \\ Universidad Autónoma de Nuevo León, Monterrey, Nuevo León, Mexico
}

\begin{abstract}
The superior semicircular canal dehiscence (SSCD) syndrome is a condition in which the lack of bone overlying the superior semicircular canal is associated with auditory and vestibular signs and symptoms. It was first described in 1998 by Minor et al., using computerized tomography (CT). It has been the diagnosis of patients that suffer from vertigo, hearing loss, chronic disequilibrium, nystagmus, tinnitus, autophony, and/or oscillopsia in the absence of other otological affections. The etiology remains unknown, but it has been concluded that it can be congenital, acquired, or a mixed syndrome, as are many other bone dehiscences. SSCD can be symptomatic or asymptomatic, and its motives are still unknown. The representative manifestation is known as Tullio's phenomenon or Hennebert's sign. The diagnosis is based on the clinical history and radiological evidence. CT is the most used study in combination with audiological and vestibular test findings. Repair through the transmastoid approach and the middle fossa approach has been used and has had the best symptomatology resolution. The latter procedure is considered the most effective, although comparative outcomes are still deficient.
\end{abstract}

Key words: Superior semicircular canal dehiscence. Vertigo disorder. Disequilibrium. Otological affection.

\section{What is known about this syndrome?}

Superior semicircular canal dehiscence (SSCD) syndrome is a condition in which the lack of bone overlying the superior semicircular canal, located in the petrotympanic region, is associated with auditory and vestibular signs and symptoms. It was first described in 1998 through two case reports by Minor et al. in 1998 using computerized tomography (CT). It has been the diagnosis of patients suffering from vertigo, hearing loss, chronic disequilibrium, nystagmus, tinnitus, autophony, and/or oscillopsia in the absence of an otological affection'.

The prevalence ranges between $3 \%$ and $8 \%$ in patients with an ear CT scan². The etiology remains unknown, but it has been concluded that it can be a mixed syndrome, congenital, or acquired, as are many other bone dehiscences ${ }^{3}$. SSCD can be symptomatic or asymptomatic, and its motives are still unknown. The representative manifestation is known as Tullio's phenomenon (nystagmus induced by loud noise) or Hennebert's sign (nystagmus induced by pressure applied to the external auditory canal). The eye movements elicited by these stimuli are aligned with the plane of the dehiscent superior semicircular canal ${ }^{4}$.

The diagnosis is based on the clinical history and radiological evidence. CT is the most used study in combination with audiological and vestibular test findings. Repair through the transmastoid approach and

\section{Correspondence:}

*Jose L. Treviño-González

E-mail: jose.trevinognz@uanl.edu.mx
Available online: 28-06-2021

Date of reception: 20-11-2020

Date of acceptance: 19-02-2021 DOI: 10.24875/RMU.20000084
Medicina Universitaria. 2021;23(2):68-69 www.medicinauniversitaria.org

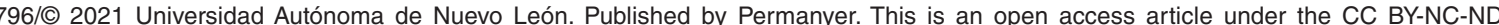
license (http://creativecommons.org/licenses/by-nc-nd/4.0/). 
the middle fossa approach has been used and has had the best symptomatology resolution. The latter procedure is considered the most effective, although comparative outcomes are still deficient ${ }^{5}$.

\section{What can we offer?}

The disease has been studied with guidelines and recommendations for diagnosis and treatment ${ }^{6}$. However, there is a lack of data related to certain aspects. Variability among prevalence has been detected due to differences in study designs, heterogeneity, and study populations, with the results changing depending on the group ${ }^{2}$. This exhibits an ambiguous and unreliable prevalence. The etiology remains unknown. Studies have failed to resolve these, with unclear conclusions of multifactorial origins ${ }^{4}$. The correlation between risk factors and SSCD is deficient. Similarly, comorbidities have yet to be associated with these types of syndromes ${ }^{5}$.

SSCD is a commonly misdiagnosed vestibular disorder due to a lack of knowledge of the pathology or its mimicking characteristics of other diseases ${ }^{6,7}$. Determining its etiology and its correlation to risk factors, comorbidities, and onset scenarios will help establish effective treatments. Future studies need to compare treatment outcomes with the pathology effectively and further research the disease's physiopathology.

\section{Ethical disclosures}

Protection of human and animal subjects. The authors declare that no experiments were performed on humans or animals for this study.

Confidentiality of data. The authors declare that they have followed the protocols of their work center on the publication of patient data.

Right to privacy and informed consent. The authors declare that no patient data appear in this article.

\section{References}

1. Nguyen T, Sheppard JP, Duong C, Ding K, Dejam D, Alkhalid Y, et al. Age and gender considerations on the symptomology in patients with superior semicircular canal dehiscence: a systematic review and case illustration. J Clin Neurosci. 2019;65:112-20.

2. Stimmer H, Hamann KF, Zeiter S, Naumann A, Rummeny EJ. Semicircular canal dehiscence in HR multislice computed tomography: distribution, frequency, and clinical relevance. Eur Arch Otorhinolaryngol. 2012;269:475-80.

3. Hernandez-Trejo AF, Cuellar-Calderon KP, Treviño-Gonzalez JL, Yamamoto-Ramos M, Campos-Coy MA, Quiroga-Garza A, et al. Prevalence of facial canal dehiscence and other bone defects by computed tomography. Eur Arch Otorhinolaryngol. 2020;277:2681-6.

4. Chilvers G, McKay-Davies I. Recent advances in superior semicircular canal dehiscence syndrome. J Laryngol Otol. 2015;129:217-25.

5. Ward BK, Carey JP, Minor LB. Superior canal dehiscence syndrome: lessons from the first 20 years. Front Neurol. 2017;8:1-10.

6. Mau C, Kamal N, Badeti S, Reddy R, Ying YL, Jyung RW, et al. Superior semicircular canal dehiscence: diagnosis and management. J Clin Neurosci. 2018;48:58-65.

7. Zhou G, Gopen Q, Poe DS. Clinical and diagnostic characterization of canal dehiscence syndrome: a great otologic mimicker. Otol Neurotol. 2007;28:920-6. 